\title{
Recipiente biodegradável e substratos para mudas de maracujazeiro
}

\author{
Mariana Santana Guerra ${ }^{1}$, Marcelo Sousa Barbosa ${ }^{1}$, Edilson $\operatorname{Costa}^{1}$, Gustavo Haralampidou \\ Costa Vieira ${ }^{1}$
}

${ }^{1}$ Universidade Estadual de Mato grosso do Sul, Unidade Universitária de Cassilândia, Cassilândia, Mato Grosso do Sul, Brasil. Email:sgmariana@hotmail.com, marcelo_sousad2@hotmail.com,mestrine@uems.br,gcv@uems.br.

Recebido: 03/05/2017; Aceito: 13/06/2017.

\section{RESUMO}

O descarte incorreto dos recipientes plásticos usados na produção de mudas causa impactos ao ambiente. O maracujazeiro amarelo é produzido em grande escala, tendo importância fármaco-terapêutico, além de ser uma frutífera de produção rápida, sendo para isso imprescindível a obtenção de mudas de qualidade. O objetivo deste trabalho foi avaliar recipientes e substratos na formação de mudas de maracujazeiro. O experimento foi conduzido num delineamento inteiramente casualizado, em esquema fatorial $2 \times 4$, sendo 2 recipientes e 4 substratos. Como recipientes foram utilizados bucha vegetal e sacos plásticos. Em cada recipiente foram testados os seguintes substratos: $30 \%$ de esterco bovino $+70 \%$ de vermiculita; $30 \%$ de Bioplant $+70 \%$ vermiculita; $30 \%$ de esterco bovino $+70 \%$ de areia; $30 \%$ de Bioplant $+70 \%$ areia. Foram avaliadas variáveis referentes a altura, diâmetro do colo, fitomassas e relações biométricas. As mudas mais vigorosas de maracujazeiro foram obtidas com o recipiente de polietileno. Para os substratos, o melhor resultado foi observado utilizando-se a mistura de esterco e vermiculita.

Palavras-chave: Bucha vegetal, esterco bovino, Bioplant.

\section{Biodegradable container and substrates for passion fruit seedlings}

\begin{abstract}
Improper disposal of plastic containers used in the production of seedlings cause impacts on the environment. The passion fruit is produced on a large scale, has importance for therapeutic drug, and is a fruit of rapid production and for this the seedlings high quality area very important. The objective of this work was to evaluate containers and substrates in the formation of passion fruit seedlings. The experiment was conducted in a completely randomized design, in a 2 x 4 factorial scheme, with 2 containers and 4 substrates. The containers were the vegetable loofah and plastic bags. In each container the following substrates were tested: $30 \%$ of cattle manure $+70 \%$ of vermiculite; $30 \%$ of Bioplant $+70 \%$ vermiculite; $30 \%$ of cattle manure $+70 \%$ sand; $30 \%$ of Bioplant $+70 \%$ sand. The experiment was conducted in a completely randomized design in a factorial $2 \times 4$, with 2 containers and 4 substrates. The height, stem diameter, dry mass and biometric relationships were evaluated. The most vigorous seedlings were obtained with polyethylene container. For the substrates, the best result was observed using the mixture of manure and vermiculite.
\end{abstract}

Key words: Vegetable loofah, cattle manure, Bioplant. 


\section{Introdução}

A produção de mudas visa a obtenção de plantas vigorosas para formação ou renovação de pomares e também busca suprir a necessidade de reposição de determinadas plantas. É necessário que as mudas tenham capacidade de adaptação e resistência às intempéries pois ao saírem do viveiro com condições protegidas, são transplantadas para o local definitivo de produção e recebem estresse fisiológico das condições ambientais. Portanto, uma muda de alta qualidade precisa se vigorosa, com pegamento e sobrevivência elevada no campo.

Para promover uma muda de alta qualidade várias tecnologias e técnicas são utilizadas, tais como: sementes selecionadas e vigorosas, substratos adequados, recipientes e ambientes protegidos compatíveis, controle da irrigação e adubação suplementar entre outras. Estudos com recipientes para formação de mudas de maracujá, envolvendo tipos, tamanhos e volumes de recipientes (VERDIAL et al., 2000; RIBEIRO et al., 2005; CHAGAS et al., 2006; COSTA et al., 2009; COSTA et al., 2010; SILVA et al., 2010; COSTA et al., 2011; ZACCHEO et al., 2013) foram realizados e forneceram informações preciosas sobre tubetes, bandejas e sacolas plásticas, contudo não foram encontrados relatos com recipientes biodegradáveis.

O uso de recipientes biodegradáveis pode auxiliar o meio ambiente sendo um produto que se degrada em menor tempo no solo quando comparado aos recipientes plásticos. Ferraz e Cereda (2010), assim como Ferraz et al. (2015), observaram que as mudas de Petúnias Comuns (petunia $x$ hybrida) se desenvolveram melhor em sacos plásticos que em recipientes biodegradáveis e que este tipo de recipiente promovia déficit hídrico em razão da alta permeabilidade. Mudas de eucaliptos apresentaram menor comprimento radicular em tubetes biodegradáveis que em tubetes plásticos, ambos com o mesmo volume (IATAURO, 2001). Tubetes biodegradáveis com metade do volume de tubetes plásticos não afetaram negativamente o crescimento do sistema radicular e a muda completa de aroeira (IATAURO, 2004).

Conti et al. (2012) verificaram que o custo das mudas das espécies Eucalyptus urophylla (S. T. Blake), Eucalyptus citriodora (Corymbia citriodora (Hook.) K.D. Hill \& L.A.S. Johnson) e Tabebuia chrysotricha (Mart. ex A. DC.) standl.) em tubete biodegradável apresentou-se $18 \%$ mais elevado que o custo no tubete de polietileno. Vichiato et al. (2008) estudando a substituição do palito de xaxim [Dicksonia sellowiana (Presl.) Hook.] por bucha vegetal (Luffa cylindrica L.) no cultivo de Dendrobium nobile Lindl verificaram que a bucha vegetal não substitui o xaxim, mas pode representar uma alternativa promissora e novos estudos devem ser realizados.

Diante do exposto e visando um sistema produtivo cada vez mais sustentável, objetivou-se estudar o uso da bucha vegetal (Luffa cylindrica) como recipiente alternativo em comparação com sacos plásticos na produção de mudas de maracujá, assim como diferentes substratos.

\section{Material e Métodos}

$\mathrm{O}$ experimento com recipientes alternativos e substratos foi conduzido na Universidade Estadual de Mato Grosso do Sul (UEMS), Unidade Universitária de Cassilândia-MS, no período de fevereiro a abril de 2015. O local possui latitude $19^{\circ} 07^{\prime} 21^{\prime \prime} \mathrm{S}$ e longitude $51^{\circ} 43^{\prime} 15^{\prime \prime}$ W e altitude de $516 \mathrm{~m}$., conduzido em delineamento inteiramente casualizado, em esquema fatorial $2 \times 4$, sendo 2 recipientes e 4 substratos, com 5 repetições contendo 4 plantas. $\mathrm{O}$ experimento foi realizado sob ambiente protegido, possuindo $8,00 \mathrm{~m}$ de largura por 18,00 m de comprimento e 4,00 $\mathrm{m}$ de altura, fechamento em $90^{\circ}$ graus com tela preta nas laterais, tela aluminizada na cobertura a $3,30 \mathrm{~m}$ e ambas as telas de $50 \%$ de sombreamento.

Foram avaliados dois recipientes: bucha vegetal com capacidade média de 1,0 litro de substrato e saquinho plástico de $15,0 \mathrm{~cm}$ x $25,0 \mathrm{~cm}$, com capacidade média de 1,8 litros que tem demonstrado as melhores mudas conforme literatura (COSTA et al., 2009; COSTA et al., 2010; COSTA et al., 2011). Foram testados 4 substratos, designados por: (S1) $30 \%$ de esterco bovino $+70 \%$ de vermiculita; (S2) 30\% de Bioplant ${ }^{\circledR}+70 \%$ vermiculita; (S3) $30 \%$ de esterco bovino $+70 \%$ de areia; (S4) $30 \%$ de Bioplant ${ }^{\circledR}+70 \%$ areia.

A semeadura foi realizada no dia 09 de fevereiro de 2015. Foram coletadas as alturas das mudas com régua milimetrada aos 15 (AP1), 30 (AP2) e 45 (AP3) dias após a semeadura (DAS). Aos 45 DAS foram mensuradas, também, o diâmetro do colo (DC) com paquímetro digital, a massa seca da parte aérea (MSPA), a massa seca do sistema radicular (MSSR). As fitomassas foram obtidas em balança analítica depois de serem secas em estufa com circulação de ar por 72 horas a $65^{\circ} \mathrm{C}$. A partir dos dados coletados foi determinada a massa seca total (MST), a relação entre a massa seca aérea e radicular (RMS), o índice de qualidade de Dckson (IQD) e a taxa de crescimento absoluto (TCA) em dois períodos, dos 15 aos 30 DAS (TCA1) e dos 30 aos 45 DAS (TCA2). A taxa de crescimento absoluto foi baseada na equação definida por Benincasa (2003). O esterco bovino foi adquirido de frigorífico, compostado por 45 dias e realizada análise da composição química (Tabela 1). 
Tabela 1. Resultado da análise da composição química do esterco bovino utilizado no experimento. Cassilândia, MS, 2015.

\begin{tabular}{|c|c|c|c|c|c|c|c|}
\hline $\mathrm{N}$ & $\mathrm{P}_{2} \mathrm{O}_{5}$ & $\mathrm{~K}_{2} \mathrm{O}$ & $\mathrm{Ca}$ & $\mathrm{Mg}$ & $S$ & $\mathrm{U}-65^{\circ} \mathrm{C}$ & $\mathrm{C}$ \\
\hline 0,9 & 0,3 & 0,1 & 0,3 & 0,1 & 0,2 & 2,0 & 11,0 \\
\hline $\mathrm{Na}$ & $\mathrm{Cu}$ & $\begin{array}{c}\mathrm{Fe} \\
-\mathrm{mg} \mathrm{kg}^{-}\end{array}$ & $\mathrm{Mn}$ & $\mathrm{Zn}$ & $\mathrm{C} / \mathrm{N}$ & $\begin{array}{c}\mathrm{pH} \\
\mathrm{CaCl}_{2}\end{array}$ & $\begin{array}{c}\mathrm{MO} \\
\%\end{array}$ \\
\hline 624 & 18 & 12103 & 204 & 53 & $12 / 1$ & 5,3 & 20,0 \\
\hline
\end{tabular}

$\left(\mathrm{N}=\right.$ nitrogênio, $\mathrm{P}_{2} \mathrm{O}_{5}=$ pentóxido de fósforo, $\mathrm{K}_{2} \mathrm{O}=$ óxido de potássio, $\mathrm{Ca}=$ cálcio, $\mathrm{Mg}=$ magnésio, $\mathrm{S}=$ enxofre, $\mathrm{U}=$ umidade, $\mathrm{C}=$ carbono, Na $=$ sódio, $\mathrm{Cu}=$ cobre, $\mathrm{Fe}=$ ferro, $\mathrm{Mn}=$ manganês, $\mathrm{Zn}=$ zinco, $\mathrm{C} / \mathrm{N}=$ relação carbono nitrogênio, $\mathrm{MO}=$ matéria orgânica).

O Bioplant ${ }^{\circledR}$ e a areia fina foram obtidas no comércio local. O Bioplant ${ }^{\circledR}$, segundo o fabricante, é composto por fibra de coco, casca de "pinus", esterco, serragem, vermiculita, casca de arroz, cinza, gesso agrícola, carbonato de cálcio, magnésio, termofosfato magnesiano (yoorin) e aditivos (fertilizantes).

Os dados foram submetidos à análise de variância (teste F) e as médias comparadas pelo teste de Tukey para os substratos e pelo teste "t" de student para os recipientes, ambos a 5\% de probabilidade, com o software Sisvar 5.3 (FERREIRA, 2010). Os dados da taxa de crescimento TCA1 e TCA2 foram transformados em raiz de $\mathrm{x}$ mais meio.

\section{Resultados e Discussão}

Não foram obervadas interações entre substratos e recipientes para a altura de planta aos 15 (AP1) e 30 (AP2) dias após a semeadura (DAS), bem como para a taxa de crescimento absoluto entre esse intervalo de tempo (TCA1). Para essas variáveis foram avaliados os fatores isolados (Tabela 2).

Aos 15 DAS a altura das mudas nos dois recipientes não diferiram. Aos 30 DAS foram verificadas maiores mudas na sacola de polietileno, assim como maior taxa de crescimento no intervalo de 15 a 30 DAS, em comparação com o recipiente alternativo biodegradável (bucha vegetal) (Tabela 2). Três aspectos podem ser obervados para o menor crescimento das mudas no recipiente alternativo, primeiro o volume do recipiente, segundo a elevada permeabilidade deste material e terceiro um possível efeito alelopatico deste material.
Por possuir maior volume e maior espaço para o crescimento das raízes, o saco de polietileno propiciou melhor condição para o crescimento das mudas e consequentemente plantas maiores (Tabela 2), resultados que corroboram com Verdial et al. (2000), Ribeiro et al. (2005), Costa et al. (2009), Costa et al. (2010) e Costa et al. (2011) que verificaram mudas de maracujazeiro maiores quando plantadas em recipientes de maior volume.

$\mathrm{Na}$ comparação dos substratos foi verificado nos substratos que continham esterco bovino ( $\mathrm{S} 1$ e S3) as maiores mudas e a maior taxa de crescimento (Tabela 2). Verificou-se, também, que na comparação entre o $S 1$ e S3, aos 30 DAS, o substrato que estava misturado com a vermiculita (S1) propiciou melhores condições físicas e químicas (Tabela 1) para a formação de mudas de maracujá do que o substrato misturado com areia (S3).

As variáveis que apresentaram interação significativa para os fatores recipientes e substratos revelaram similar resultado com as variáveis sem interação significativa em que o recipiente com maior volume (R1) e os substratos com esterco bovino ( $\mathrm{S} 1 \mathrm{e}$ S3) propiciaram melhores condições de crescimento, diâmetro, acúmulo de fitomassa e qualidade de mudas (Tabela 3). Foi observado, também, que nos substratos que continham esterco bovino, aquele que estava misturado com vermiculita propiciou melhores condições físicas e químicas (Tabela 1) para a formação de mudas de maracujá que o misturado com areia apresentando maior fitomassa, diâmetro e crescimento (Tabela 3).

Tabela 2. Altura de planta aos 15 (AP1), 30 (AP2) dias após a semeadura (DAS) e taxa de crescimento absoluto entre o intervalo de tempo de 15 e 30 DAS (TCA1) para os fatores isolados recipientes (R) e subtratos (S). Cassilândia, 2015.

\begin{tabular}{lccc}
\hline & AP1 $(\mathrm{cm})$ & AP2 $(\mathrm{cm})$ & TCA1 $(\mathrm{cm} /$ dia $)$ \\
\hline R1: Saco de polietileno 15,0 x 25,0 cm, 1,8 $\mathrm{L}$ & $3,18 \mathrm{a}^{*}$ & $4,86 \mathrm{a}^{*}$ & $0,11 \mathrm{a}^{*}$ \\
R2: Recipiente alternativo, bucha vegetal & $3,02 \mathrm{a}$ & $4,09 \mathrm{~b}$ & $0,07 \mathrm{~b}$ \\
\hline S1: $30 \%$ de esterco bovino $+70 \%$ de vermiculita & $3,58 \mathrm{a}^{*}$ & $6,32 \mathrm{a}^{*}$ & $0,18 \mathrm{a}^{*}$ \\
S2: $30 \%$ de Bioplant & ${ }^{\circledR}+70 \%$ vermiculita & $3,02 \mathrm{~b}$ & $0,03 \mathrm{~b}$ \\
S3: $30 \%$ de esterco bovino $+70 \%$ de areia & $3,12 \mathrm{ab}$ & $5,05 \mathrm{~b}$ & $0,13 \mathrm{a}$ \\
S4: $30 \%$ de Bioplant $^{\circledR}+70 \%$ areia & $2,67 \mathrm{~b}$ & $3,1 \mathrm{c}$ & $0,03 \mathrm{~b}$ \\
\hline CV $(\%)$ & 12,45 & 20,34 & 5,11 \\
\hline
\end{tabular}

*Letras iguais minúsculas nas colunas, para cada parâmetro e cada fator, não diferem entre si pelo Teste de Tukey para os substratos e pelo teste $\mathrm{t}$ para os recipientes, a $5 \%$ de probabilidade. 
Tabela 3. Interação entre recipiente (R) e substratos (S) para altura de planta aos 45 DAS (AP3), diâmetro do colo (DC), taxa de crescimento entre os intervalos de tempo de 30 a 45 DAS (TCA2), relação entre a fitomassa seca da parte aérea e do sistema radicular (RMS), fitomassa seca da parte aérea (MSPA), fitomassa seca do sistema radicular (MSSR), fitomassa seca total (MST) e índice de qualidade e Dickson (IQD). Cassilândia, 2015.

\begin{tabular}{|c|c|c|c|c|c|c|c|c|}
\hline & $\mathrm{R} 1$ & R2 & R1 & $\mathrm{R} 2$ & R1 & $\mathrm{R} 2$ & R1 & $\mathrm{R} 2$ \\
\hline & \multicolumn{3}{|c|}{$\mathrm{AP} 3(\mathrm{~cm})$} & $\mathrm{DC}(\mathrm{mm})$ & \multicolumn{2}{|c|}{ TCA2 (cm/dia) } & \multicolumn{2}{|c|}{ RMS } \\
\hline $\mathrm{S} 1$ & $41,82 \mathrm{aA}^{*}$ & $10,63 \mathrm{bA}$ & $4,52 \mathrm{aA}^{*}$ & $2,32 \mathrm{bA}$ & $2,30 \mathrm{aA} *$ & $0,35 \mathrm{bA}$ & $1,70 \mathrm{aA}^{*}$ & $1,13 \mathrm{bA}$ \\
\hline $\mathrm{S} 2$ & $3,99 \mathrm{aC}$ & $4,05 \mathrm{aA}$ & $1,04 \mathrm{aC}$ & $0,74 \mathrm{aC}$ & $0,02 \mathrm{aC}$ & $0,05 \mathrm{aA}$ & $1,36 \mathrm{aA}$ & $1,10 \mathrm{aA}$ \\
\hline S3 & $19,15 \mathrm{aB}$ & $8,94 \mathrm{bA}$ & $2,65 \mathrm{aB}$ & $1,68 \mathrm{bAB}$ & $0,91 \mathrm{aB}$ & $0,29 \mathrm{bA}$ & $1,29 \mathrm{aA}$ & $1,52 \mathrm{aA}$ \\
\hline S4 & $3,3 \mathrm{aC}$ & $3,84 \mathrm{aA}$ & $1,03 \mathrm{aC}$ & $1,00 \mathrm{aBC}$ & $0,02 \mathrm{aC}$ & $0,04 \mathrm{aA}$ & $0,82 \mathrm{bB}$ & $1,23 \mathrm{aA}$ \\
\hline \multirow[t]{2}{*}{$\mathrm{CV}(\%)$} & 39,87 & & 24,07 & & 11,90 & & 20,90 & \\
\hline & & \multicolumn{2}{|c|}{ MSPA } & MSSR & & $\overline{\mathrm{MST}}$ & \multicolumn{2}{|c|}{ IQD } \\
\hline S1 & $4,68 \mathrm{aA}^{*}$ & $1,35 \mathrm{bA}$ & $2,79 \mathrm{aA}^{*}$ & $1,19 \mathrm{bA}$ & $7,47 \mathrm{aA}^{*}$ & $2,54 \mathrm{bA}$ & $0,69 \mathrm{aA}^{*}$ & $0,44 \mathrm{bA}$ \\
\hline S2 & $1,15 \mathrm{aC}$ & $0,42 \mathrm{bB}$ & $0,83 \mathrm{aD}$ & $0,38 \mathrm{bB}$ & $1,99 \mathrm{aC}$ & $0,80 \mathrm{bB}$ & $0,38 \mathrm{aB}$ & $0,12 \mathrm{bB}$ \\
\hline S3 & $2,40 \mathrm{aB}$ & $2,03 \mathrm{aA}$ & $1,92 \mathrm{aB}$ & $1,37 \mathrm{bA}$ & $4,32 \mathrm{aB}$ & $3,40 \mathrm{bA}$ & $0,55 \mathrm{aAB}$ & $0,51 \mathrm{aA}$ \\
\hline S4 & $1,08 \mathrm{aC}$ & $0,62 \mathrm{aB}$ & $1,33 \mathrm{aC}$ & $0,50 \mathrm{bB}$ & $2,41 \mathrm{aC}$ & $1,13 \mathrm{bB}$ & $0,60 \mathrm{aA}$ & $0,22 \mathrm{bB}$ \\
\hline $\mathrm{CV}(\%)$ & 23,55 & & 24,08 & & 19,77 & & 25,28 & \\
\hline
\end{tabular}

*Letras iguais maiúsculas nas colunas e minúsculas nas linhas, para cada parâmetro, não diferem entre si pelo Teste de Tukey para os substratos e pelo teste t para os recipientes, a 5\% de probabilidade. $\mathrm{R} 1=$ Saco de polietileno 15,0 x 25,0 cm, 1,8 L; R2 = Recipiente alternativo, bucha vegetal. $\mathrm{S} 1$ $=30 \%$ de esterco bovino $+70 \%$ de vermiculita; $\mathrm{S} 2=30 \%$ de Bioplant ${ }^{\circledR}+70 \%$ vermiculita; $\mathrm{S} 3=30 \%$ de esterco bovino $+70 \%$ de areia; $\mathrm{S} 4=30 \%$ de Bioplant ${ }^{\circledR}+70 \%$ areia.

Aos 25 e 30 DAS verificou-se que as maiores plantas assim como a maior taxa de crescimento absoluto se deu nos substratos que continham esterco bovino com vermiculita em relação à areia (Tabela 2), conforme verificados também nas variáveis de crescimento, diâmetro, fitomassas e de qualidade (Tabela 3). Observou-se no crescimento das mudas que a associação de esterco bovino e vermiculita foram determinantes para obtenção de plantas maiores. A vermiculita melhorou as condições do substrato pois liberou íons de magnésio para a solução e absorveu fósforo e nitrogênio na forma amoniacal (TÚLLIO JÙNIOR et al., 1986), desta maneira este material propiciou condições mais favoráveis ao crescimento das mudas de maracujazeiro. Estes resultados com a vermiculita estão de acordo com Minami (2000), Negreiros et al. (2004) e Costa et al. (2009) onde ressaltam a grande capacidade de aeração e retenção de água da vermiculita propiciando assim melhor desenvolvimento da muda.

Nos substratos que continham Bioplant ${ }^{\circledR}$ foram verificadas as menores plantas e as menores taxas de crescimento no período de 15 a 30 DAS (Tabela 2), assim como nas variáveis de diâmetro, fitomassas e de qualidade (Tabela 3). Mesmo apresentando diversos materiais em sua composição como fibra de coco, casca de pinus, esterco, serragem, vermiculita, casca de arroz, cinza, gesso agrícola, carbonato de cálcio, magnésio, termofosfato magnesiano (yoorin) e aditivos (fertilizantes), o Bioplanti ${ }^{\circledR}$ não propiciou condição satisfatória de crescimento das mudas de marcaujá. Para mudas florestais o Bioplant ${ }^{\circledR}$ não tem proporcionado crescimento adequado das mudas, como por exemplo para a Cratevatapia L (ALVES et al., 2012), para a Eugenia calycina (BORGES et al., 2016) e Copaíba
(DUTRA et al., 2012), similar ao ocorrido no presente estudo com o maracujazeiro.

A bucha vegetal possuía em média 1,0 litro de substrato, enquanto o saquinho plástico 1,8 litros. $\mathrm{O}$ menor volume disponibilizou menor espaço de crescimento do sistema radicular e menor quantidade de nutrientes, consequentemente plantas menores. $\mathrm{Na}$ comparação de recipientes para mudas de maracujazeiro, Verdial et al. (2000), Ribeiro et al. (2005), Costa et al. (2009), Costa et al. (2010), Costa et al. (2011) verificaram que recipientes de menores volumes propiciaram menor crescimento.

A elevada permeabilidade da bucha vegetal poderia estar carreando os nutrientes e diminuindo a quantidade disponível para as mudas, fator que contribui para mudas de qualidade inferior. Outros experimentos com recipientes alternativos mostraram que os recipientes biodegradáveis não propiciaram condições adequadas às mudas, como por exemplo, para mudas de petúnias (FERRAZ; CEREDA, 2010; FERRAZ et al., 2015), de eucalipto (IATAURO, 2001) e aroeira (IATAURO, 2004). Para mudas de Dendrobium nobile Lindl, Vichiato et al. (2008) verificaram que a bucha vegetal (Luffa cylindrica) não foi favorável ao seu desenvolvimento, quando comparada ao xaxim.

\section{Conclusões}

O recipiente composto por polietileno foi mais adequado para produção de mudas quando comparado ao recipiente alternativo estudado;

O substrato composto por esterco e vermiculita se mostrou mais adequado à produção de mudas do maracujazeiro. 


\section{Agradecimentos}

À Coordenação de Aperfeiçoamento de Pessoal de Nível Superior (CAPES), pela concessão da bolsa. Ao CNPq Proc. No 300829/2012-4 bolsa de produtividade; À FUNDECT PPP (Programa Primeiros Projetos) 05/2011 Proc. No 23/200.647/2012. À FUNDECT/UEMS pela bolsa de Iniciação Científica.

\section{Referências Bibliográficas}

ALVES, E. U.; MOURA, S. S. S.; MOURA, M. F.; GUEDES, R. S.; ESTRELA, F. A. Germinação e vigor de sementes de Crateavatapia L. em diferentes substratos e temperaturas. Revista Brasileira de Fruticultura, Jaboticabal-SP, v. 34, n. 4, p. 1208-1215, 2012.

BENINCASA, M. M. P. Análise de crescimento de plantas: noções básicas. Jaboticabal-SP: FUNEP, 2003. 41 p

BORGES, K. C. F.; SANTANA, D. G.; LOPES, S. W.; PEREIRA, V. J. Coloração do fruto e substrato na emergência e no crescimento de plantas de Eugenia calycinas Cambess. Floresta e Ambiente, Seropédica-RJ, v. 23, n. 4, p. 544-554 2016.

CHAGAS, I. M.; TAVARES, J. C.; FREITAS, R. S.; RODRIGUES, G. S. O. Formação de mudas de maracujá amarelo em quatro tamanhos de recipiente. Revista Verde, Pombal-PB, v.1, n. 2, p. 122-133, 2006.

CONTI, A. C.; REIS, R. C. S.; CONTI, C.; DANIEL NETO, R. F.; ARANTES, A. K. Análise do desenvolvimento e da viabilidade econômica do plantio de mudas de árvores em tubetes biodegradáveis. RETEC, Ourinhos-SP, v. 05, n. 01, p. 113-121, 2012

COSTA E.; RODRIGUES E. T.; ALVES V. B.; SANTOS, L. C. R.; VIEIRA L. C. R. Efeitos da ambiência, recipientes e substratos no desenvolvimento de mudas de maracujazeiroamarelo em Aquidauana - MS. Revista Brasileira de Fruticultura, Jaboticabal-SP, v. 31, n. 1, p. 236-244, 2009.

COSTA, E.; LEAL, P. A. M.; SANTOS, L. C. R.; VIEIRA, L. C. R. Ambientes de cultivo, recipientes e substratos na produção de biomassa foliar e radicular em mudas de maracujazeiro amarelo em Aquidauana - MS. Ciência e Agrotecnologia, Lavras-MG, v. 34, n. 2, p. 461-467, 2010

COSTA, E.; SANTOS, L. C. R.; CARVALHO, C.; LEAL, P. A. M.; GOMES, V. A. Volumes de substratos comerciais, solo e composto orgânico afetando a formação de mudas de maracujazeiro-amarelo em diferentes ambientes de cultivo. Revista Ceres, Viçosa-MG, v. 58, n. 2, p. 216-222, 2011.

DUTRA, T. R.; GRAZZIOTTI, P. H.; SANTANA, R. C; MASSAD, M. D. Desenvolvimento inicial de mudas de copaíba sob diferentes níveis de sombreamento e substratos. Revista Ciência Agronômica, Fortaleza-CE, v. 43, n. 2, p. 321-329, 2012.

FERRAZ, M. V.; CEREDA, M. P. Determinação das características morfológica de petúnias comuns (petunia $x$ hybrida) cultivadas em tubetes biodegradáveis. Scientia Agraria Paranaensis, Marechal Cândido Rondon-PR, v. 9, n. 1, p. 94-107, 2010.
FERRAZ, M. V.; CEREDA, M. P.; IATAURO, R. A. produção de mudas de petúnia comum em tubetes biodegradáveis em substituição aos sacos plásticos. Brazilian Journal of Biosystems Engineering, Tupã-SP, v. 9, n. 1, p. 74-83, 2015

FERREIRA, D. F. Sisvar - Sistema de análise de variância. Versão 5.3. Lavras-MG: UFLA. 2010.

IATAURO, R. A. Avaliação de tubetes biodegradáveis para a produção e o acondicionamento de mudas de Eucalyptus grandis Hill ex. Maiden. 2001. 33 p. Monografia de Trabalho de Conclusão de Curso (Graduação em Agronomia) - Instituto de Biociências de Botucatu, Universidade Estadual Paulista, Botucatu-SP, 2001.

IATAURO, R. A. Avaliação energética da substituição de tubetes de plástico por tubetes biodegradáveis na produção de mudas de aroeira-Schinus terebinthifolius Raddi. 2004. 59 p. Dissertação (Mestrado em Agronomia) Faculdade de Ciências Agronômicas, UNESP, Botucatu-SP, 2004.

MINAMI, K. A. A pesquisa em substrato no Brasil. In: KÄMPF, N. A.; FERMINO, M. H. Substratos para plantas: a base da produção vegetal em recipientes. Porto Alegre-RS: Gênesis, 2000. p. 159-162.

NEGREIROS, J. R. S.; ÁlVARES, V. S.; BRAGA, L. R.; BRUCKNER, C. H. Diferentes substratos na formação de mudas de maracujazeiro-amarelo. Revista Ceres, Viçosa-MG, v. 51, n. 294, p. 243-249, 2004.

RIBEIRO, M. C. C.; MORAIS, M. J. A.; SOUSA, A. H.; LINHARES, P. C. F.; BARROS JUNIOR, A. P. Produção de mudas de maracujá-amarelo com diferentes substratos e recipientes. Caatinga, Mossoró-RN, v. 18, n. 3, p.155-158, 2005

SILVA, E. A.; MARUYAMA, W. I.; MENDONÇA, V.; FRANCISCO, M. G. S.; BARDIVIESSO, D. M.; TOSTA, M. S. Composição de substratos e tamanho de recipientes na produção e qualidade das mudas de maracujazeiro 'amarelo'. Ciência e Agrotecnologia, Lavras-MG, v. 34 , n. 3, p. 588$595,2010$.

TÚLLIO JÚNIOR, A. A.; NOGUEIRA, R. R.; MINAMI, K. Uso de diferentes substratos na germinação e formação de mudas de pimentão (Capsicumannum L.). O Solo, ViçosaMG, v. 78, n. 1, p. 15-18, 1986.

VERDIAL, M. F, L., SANTOS, M; TESSARIOLI NETO, J. Métodos de formação de mudas de maracujazeiro amarelo. Scentia agricola, Piracicaba-SP, v. 57, n. 4, p. 795-798, 2000.

VICHIATO, M. R. M.; VICHIATO, M.; CASTRO, D. M.; DUTRA, L. F.; PASQUAL, M. ARAÚJO, T. S. Bucha vegetal e fertilização organo-mineral no cultivo de Dendrobiumnobile Lindl. Revista da FZVA, Uruguaiana-RS, v. 15, n. 1, p. 3442, 2008.

ZACCHEO, P. V. C.; AGUIAR, R. S.; STENZEL, N. M. C.; NEVES, C. S. V. J. Tamanho de recipientes e tempo de formação de mudas no desenvolvimento e produção de maracujazeiro-amarelo. Revista Brasileira de Fruticultura, Jaboticabal-SP, v. 35, n. 2, p. 603-607, 2013. 